Research Paper

\title{
Prognostic Model to Predict Survival Outcome for Curatively Resected Liposarcoma: A Multi-Institutional Experience
}

\author{
Yoon Jung $\mathrm{Oh}^{1 *}$, Seong Yoon $\mathrm{Yi}^{2 *}$, Ki Hyang $\mathrm{Kim}^{3}$, Yong Jin $\mathrm{Cho}^{4}$, Seung Hoon Beum${ }^{5}$, Young Han Lee', \\ Jin-Suck Suh6, Hyuk Hur7, Kyung Sik Kim7, Sung Hoon Kim ${ }^{8}$, Young Deuk Choi ${ }^{9}$, Kyoo-Ho Shin ${ }^{4}$, Hyun \\ Jung Jun ${ }^{10}$, Sung Joo Kim¹, Jeeyun Lee ${ }^{12}$, Se Hoon Park ${ }^{12}$, Sung Hoon Noh', Sun Young Rha5, and Hyo \\ Song $\mathrm{Kim}^{5}$ \\ 1. Department of Internal Medicine, Yonsei Cancer Center, Yonsei University College of Medicine, Seoul, Korea; \\ 2. Division of Hematology-Oncology, Department of Internal Medicine, Inje University Ilsan Paik Hospital; Ilsan, Korea; \\ 3. Department of Internal Medicine, Busan Paik Hospital, Inje University College of Medicine, Busan, Korea; \\ 4. Department of Orthopedic Surgery, Yonsei University College of Medicine, Seoul, Korea; \\ 5. Division of Medical Oncology, Department of Internal Medicine, Yonsei University College of Medicine, Seoul, Korea; \\ 6. Department of Radiology, Yonsei University College of Medicine, Seoul, Korea; \\ 7. Department of Surgery, Yonsei University College of Medicine, Seoul, Korea; \\ 8. Department of Obstetrics and Gynecology, Yonsei University College of Medicine, Seoul, Korea; \\ 9. Department of Urology, Yonsei University College of Medicine, Seoul, Korea; \\ 10. Division of Medical Oncology \& Hematology, Department of Internal Medicine, Seoul Medical Center, Seoul, Korea; \\ 11. Department of Surgery, Samsung Medical Center, Sungkyunkwan University School of Medicine, Seoul, Korea; \\ 12. Division of Hematology-Oncology, Department of Medicine, Samsung Medical Center, Sungkyunkwan University School of Medicine, Seoul, Korea. \\ *Yoon Jung Oh and Seong Yoon Yi equally contributed to this work as first authors. \\ $\triangle$ Corresponding author: Hyo Song Kim, M.D., Ph.D. Yonsei Cancer Center, Yonsei University Health System, 50 Yonsei-ro, Seodaemun-gu Seoul, Republic of \\ Korea, 120-752.
}

(C) Ivyspring International Publisher. Reproduction is permitted for personal, noncommercial use, provided that the article is in whole, unmodified, and properly cited. See http://ivyspring.com/terms for terms and conditions.

Received: 2016.02.12; Accepted: 2016.04.26; Published: 2016.06.07

\begin{abstract}
Purpose: We performed this study to analyze the clinical features and prognosis of Korean patients with liposarcoma.

Patients \& Methods: Between October 1986 and April 2013, 231 patients who were diagnosed with liposarcoma by histologic examination were enrolled in this study.

Results: The distribution of histologic subtypes was well-differentiated $(n=97,42 \%)$, myxoid $(n=$ $74,32 \%)$, dedifferentiated $(n=32,13.9 \%)$, pleomorphic $(n=15,6.5 \%)$, and round-cell liposarcoma $(n=13,5.6 \%)$. The majority of liposarcomas were located in the lower extremities $(35.5 \%)$ and retroperitoneum (34.2\%). Prognosis was worse for the trunk group compared with the extremity group (median disease-free survival [DFS] 3.3 vs. 9.9 years, respectively, $\mathrm{P}<0.001$ ). Median DFS was significantly worse in patients with high grade histology compared to those with low grade histology $(16.9 \%$ vs. $65.7 \%, P<0.001)$. The independent prognostic factors associated with survival were histology (hazard ratio [HR] 3.01; 95\% confidence interval [Cl], 1.82-4.97; $\mathrm{P}<0.001$ ) and primary site $(\mathrm{HR} 1.80 ; 95 \% \mathrm{Cl}, 1.12-2.89 ; \mathrm{P}=0.015)$. Three risk groups with different survival outcomes were identified: group $1(n=98)$, no risk factors; group $2(n=92)$, one risk factor; and group $3(n=41)$, two risk factors.

Conclusions: Histologic subtype and primary site were independent prognostic factors for curatively resected liposarcoma. A prognostic model for patients with liposarcoma clarified distinct groups of patients with good prognostic discrimination.
\end{abstract}

Key words: Liposarcoma, Asian, Prognostic model, Survival. 


\section{Introduction}

Liposarcoma is the one of the most common soft tissue sarcomas (STS), consisting of over 50 different malignancies of mesenchymal origin [1, 2]. In adults, liposarcoma is the most common STS with an estimated incidence of 17-25\% [3]. It predominantly occurs in the 5th to 6th decade of life, with a slight predominance in males. The majority of cases arise in the extremities, and one third of cases arise in the visceral space $[1,3]$. Although surgical resection is the mainstay treatment for localized disease, many liposarcomas progress to advanced disease that is either unresectable, metastatic, or both. For advanced cases, the mortality rate is high and both local and systemic tumor burden can cause significant morbidity $[4,5]$.

Liposarcoma is divided into 5 subtypes based on Evans Classification: well-differentiated, myxoid, dedifferentiated, round, and pleomorphic [6]. The subtype is characterized by distinct genetic or molecular aberrations and clinical outcome $[7,8]$. Well-differentiated and myxoid liposarcoma are low-grade tumors, whereas dedifferentiated, round-cell, and pleomorphic liposarcoma are high-grade tumors. Low-grade histology depicts a low frequency of metastasis and high-grade tumors often manifest with clinically aggressive behavior resulting in distant metastasis [9-11]. However, despite the heterogeneity of liposarcoma, most previous studies have depicted only a subset of liposarcoma cases.

In this study, we analyzed a large population of patients who underwent curative resection for liposarcoma. We analyzed the clinicopathologic features and outcomes in these patients. We further explored independent prognostic factors and devised a prognostic model to facilitate decision making in clinical practice.

\section{Methods}

\section{Patients}

Between October 1986 and April 2013, 231 patients, who were diagnosed with liposarcoma by histologic examination, at Yonsei University Health System, Samsung Medical Center, Ilsan Paik Hospital, Seoul Medical Center and Busan Paik Hospital were enrolled. The criteria for inclusion were as follows: (1) pathologically confirmed diagnosis of liposarcoma; (2) a complete set of clinical information, which was defined by patient demographics, primary tumor site, stage, treatment record, and vital status; and (3) the absence of distant metastasis.

Liposarcomas were classified into 5 histologic subgroups based on Evans Classification [6] as well-differentiated, myxoid, dedifferentiated, round, and pleomorphic. The primary lesion sites were categorized into 7 groups including the lower extremities, upper extremities, thoracic cavity, trunk, retroperitoneum, inguinal area, and the head and neck. A primary site was considered as being in the upper extremities when it was at or beyond the shoulder joint, and as the lower extremities when it was located in the thigh or leg. Retroperitoneal tumors were defined, according to the surgical record, as whether any of the following resections were performed (colon, small bowel, pancreas, spleen, bladder, and uterus). Tumor burden was determined by the maximum diameter of the primary tumor at the time of surgery. The resection margin was determined by gross and microscopic examinations and a negative margin was defined as when there was no evidence of tumor cells within $\geq 1 \mathrm{~mm}$ from the edge of the specimen. Administration of postoperative adjuvant radiotherapy or chemotherapy was recorded. This study was carried out in accordance with the Declaration of Helsinki and approved by the Institutional Review Board. Patient information was de-identified and IRB waived informed consent from study subjects.

\section{Statistical analyses}

The primary end-point was to assess survival outcome using the Kaplan-Meier method. The disease-free survival (DFS) was defined as from the time of surgery to initial tumor relapse or death. Overall survival (OS) was defined as from the date of diagnosis to date of death related to the disease or complications. Duration of follow up was defined from the time of diagnosis to death. In the case of death, it was classified as an "event" and, in the case of loss of follow up, it was censoring up to that time. Survival rates were compared using the log-rank test. OS and DFS were assessed with respect to following factors: age, sex, histologic subtype, resection margin (negative or positive), primary site, tumor burden, and adjuvant treatment. Multivariate analysis was performed using stepwise Cox proportional hazards regression modeling. Two-sided P-values $\leq 0.05$ were considered statistically significant.

\section{Results}

\section{Patient characteristics}

Two-hundred and thirty-one patients who underwent surgical resection were analyzed. The clinical characteristics of the enrolled patients are presented in Table 1. There were 90 females and 141 males with median age of 55 years (range, 18-90). Median tumor size was $13 \mathrm{~cm}$ (range, 1-48), and 
approximately two-thirds of cases $(61.9 \%)$ had negative resection margins. The majority of liposarcomas were located in the lower extremities $(35.5 \%)$ and retroperitoneum (34.2\%).

The distributions of histologic subtypes were as follows: well-differentiated $(\mathrm{n}=97,42 \%)$, myxoid $(\mathrm{n}=$ $74,32 \%)$, dedifferentiated $(\mathrm{n}=32,13.9 \%)$, pleomorphic $(\mathrm{n}=15,6.5 \%)$, and round-cell $(\mathrm{n}=13$, $5.6 \%$ ), respectively. Patients were dichotomized into low (well-differentiated and myxoid types) and high-grade groups (dedifferentiated, round, and pleomorphic types) according to histologic type. There were 171 (74.0\%) patients with low-grade and $60(26 \%)$ patients with high-grade liposarcoma.

Adjuvant therapy was administered in 82 patients (35.5\%), of which $71(86.6 \%)$ and 15 (18.3\%) received radiotherapy or chemotherapy, respectively. The adjuvant treatment was introduced in $29.2 \%$ of patients with low-grade and $53.3 \%$ of those with high-grade liposarcoma, respectively. High-grade tumors were significantly associated with older age $(\mathrm{P}$ $=0.02)$, retroperitoneal location $(\mathrm{P}=0.01)$, positive resection margins $(\mathrm{P}<0.01)$, and adjuvant treatment $(\mathrm{P}$ $<0.01$ ).

\section{Survival analyses}

With a median follow-up time of 3.38 years, the 5 -year DFS and OS rates for all patients were 54.5\% and $75.1 \%$, respectively. The 5-year DFS according to histologic subtypes were $67.8 \%, 63.5 \%, 22.1 \%, 25.2 \%$ and $20.5 \%$ for well-differentiated, myxoid, dedifferentiated, pleomorphic, and round-cell liposarcoma, respectively (Table 2). The 5-year OS according to histologic subtypes were $87.1 \%, 79.4 \%$, $49.0 \%, 50.0 \%, 42.3 \%$ for well-differentiated, myxoid, dedifferentiated, pleomorphic, and round-cell liposarcoma, respectively. Kaplan-Meier analysis revealed that DFS was significantly worse in patients with high-grade histology compared to those with low-grade histology (16.9\% vs. $65.7 \%, \mathrm{P}<0.001$, Figure $1 \mathrm{~A})$. The 5-year OS rates for high and low-grade tumors were $47.8 \%$ and $83.5 \%$, respectively ( $\mathrm{P}<0.001$, Figure 1B). The most favorable prognoses were observed in patients with liposarcoma located in the inguinal/genitalia regions $(80.8 \%)$ and the extremities $(64.8 \%$ for the upper extremities and $77.8 \%$ for the lower extremities), whereas the prognoses were reduced in those with liposarcoma located in the trunk (including the retroperitoneum), intra-abdomen, and thoracic cavity $(41.8 \%, 46.4 \%$, and $40.1 \%$, respectively; $\mathrm{P}=0.002$, Figure $2 \mathrm{~A}$ ). For the primary lesion sites, prognoses were worse for the trunk group (thoracic cavity, retroperitoneum and intra-abdomen) compared with the extremities group (inguinal/genitalia, upper/lower extremity and head/neck) with a median 5-year DFS of 3.3 and 9.9 years, respectively $(\mathrm{P}<0.001$, Figure $2 \mathrm{C})$. Similar patterns were noted for the OS; 5-year OS was significantly worse in the trunk group compared with the extremities groups (median OS, 10.9 vs.15.2 years; $\mathrm{P}<0.001$; Figures 2B and 2D).

Table 1. Patient characteristics.

\begin{tabular}{|c|c|c|c|c|}
\hline Variables & $\begin{array}{l}\text { Total }(\mathrm{N}=231, \\
\%)\end{array}$ & Low $(\mathrm{n}=171)$ & High $(n=60)$ & $P$ value \\
\hline Age (Median) & $55(18-90)$ & $53(18-88)$ & $60(22-90)$ & 0.02 \\
\hline Gender & & & & 0.54 \\
\hline Male & $141(61.0)$ & $102(59.6)$ & $39(65.0)$ & \\
\hline Female & $90(39.0)$ & $69(40.4)$ & $21(35.0)$ & \\
\hline \multicolumn{5}{|l|}{ Histologic variant } \\
\hline Well differentiated & $97(42.0)$ & - & - & \\
\hline Myxoid & $74(32.0)$ & - & - & \\
\hline Dedifferentiated & 32 (13.9) & - & - & \\
\hline Pleomorphic & $15(6.5)$ & - & - & \\
\hline Round & $13(5.6)$ & - & - & \\
\hline Primary site & & & & 0.01 \\
\hline Lower extremity & $82(35.5)$ & $68(39.8)$ & $14(23.3)$ & \\
\hline Retroperitoneum & $79(34.2)$ & $54(31.6)$ & $25(41.7)$ & \\
\hline Thoracic cavity & $20(8.7)$ & $11(6.4)$ & $9(15.0)$ & \\
\hline Intra-abdominal & $15(6.5)$ & $8(4.7)$ & 7 (11.7) & \\
\hline Inguinal and genital & $14(6.0)$ & $12(7.0)$ & $2(3.3)$ & \\
\hline Upper extremity & $11(4.8)$ & $9(5.3)$ & $2(3.3)$ & \\
\hline Head and Neck & $10(4.3)$ & $9(5.2)$ & $1(1.7)$ & \\
\hline \multicolumn{5}{|l|}{ Tumor burden } \\
\hline Median (cm, range) & $13(1-48)$ & $13(1-48)$ & $13.5(2-36)$ & 0.76 \\
\hline Margin & & & & $<0.01$ \\
\hline Negative & $143(61.9)$ & $117(68.4)$ & $26(43.3)$ & \\
\hline Positive & $88(38.1)$ & $54(31.6)$ & $34(56.7)$ & \\
\hline Adjuvant treatment & & & & $<0.01$ \\
\hline No treatment & $149(64.5)$ & $121(70.8)$ & $28(46.7)$ & \\
\hline Radiotherapy & $71(30.7)$ & $44(25.7)$ & $27(45.0)$ & \\
\hline Chemotherapy & $15(6.5)$ & $7(4.1)$ & 8 (13.3) & \\
\hline
\end{tabular}

Table 2. Analysis of Histologic Subtypes in Survival Outcome.

\begin{tabular}{lllll}
\hline Histologic Variant & Total & $\begin{array}{l}\text { 5-Year } \\
\text { DFS(\%) }\end{array}$ & 5-Year OS(\%) & $P$ \\
\hline Well differentiated & 97 & 67.8 & 87.1 & $\mathrm{P}<0.001$ \\
Myxoid & 74 & 63.5 & 79.4 & \\
Dedifferentiated & 32 & 22.1 & 49.0 & \\
Pleomorphic & 15 & 25.2 & 50.0 & \\
Round & 13 & 20.5 & 42.3 & \\
\hline
\end{tabular}

\section{A prognostic model for patients with liposarcoma}

According to univariate analyses, sex, age, resection margin, tumor diameter, histologic subtype, and primary lesion site were associated with DFS and OS. In the multivariate Cox proportional model, histologic subtype and primary site were significant independent risk factors for DFS and OS (Table 3). Among them, high-grade liposarcoma was significantly associated with a shorter DFS (hazard ratio [HR] 3.01; 95\% confidence interval [CI], 
1.82-4.97; $\mathrm{P}<0.001$, Table 3). In addition, liposarcoma located in the trunk was a significantly poor prognostic factor for DFS (HR 1.80; 95\% CI, 1.12-2.89; $\mathrm{P}=0.015)$. However, there were no significant associations with tumor relapse and mortality in terms of median tumor diameter, sex, age, adjuvant treatment, or resection margin status. Similar relationships were noted for OS (Table 3).
Table 3. Prognostic factors for overall and disease free survival in study subjects.

\begin{tabular}{|c|c|c|c|c|}
\hline Variables & DFS & & OS & \\
\hline & HR (95\% CI) & $P$ & HR $(95 \%$ CI $)$ & $P$ \\
\hline $\begin{array}{l}\text { Histologic subtype } \\
\text { (High vs Low) }\end{array}$ & $3.01(1.82-4.97)$ & $<0.001$ & $3.22(1.74-5.94)$ & $<0.001$ \\
\hline $\begin{array}{l}\text { Primary site } \\
\text { (Trunk vs Extremity) }\end{array}$ & 1. & 0.015 & .77) & 0.029 \\
\hline $\begin{array}{l}\text { Margin status } \\
\text { (negative } v \text { s positive) }\end{array}$ & $0.99(0.60$ & 0.958 & $1.09(0.59-2.01)$ & 0.795 \\
\hline $\begin{array}{l}\text { Tumor burden } \\
(\leq 13 \mathrm{~cm} v s>13 \mathrm{~cm})\end{array}$ & $1.04(0.64-1.68)$ & 0.874 & $1.25(0.65-2.38)$ & 0.507 \\
\hline Age $(\leq 60 \mathrm{yr}$ vs $>60 \mathrm{yr})$ & $1.19(0.76-1.88)$ & 0.450 & $1.52(0.85-2.71)$ & 0.158 \\
\hline Sex (Male) & $1.17(0.75-1.82)$ & 0.480 & $1.05(0.59-1.88)$ & 0.869 \\
\hline Adjuvant Tx. & $1.29(0.82-2.04)$ & 0.270 & $1.31(0.73-2.35)$ & 0.361 \\
\hline
\end{tabular}

(A)

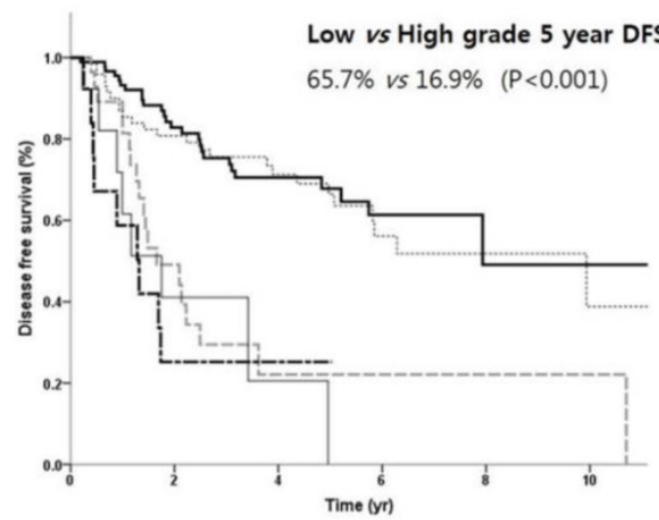

(B)

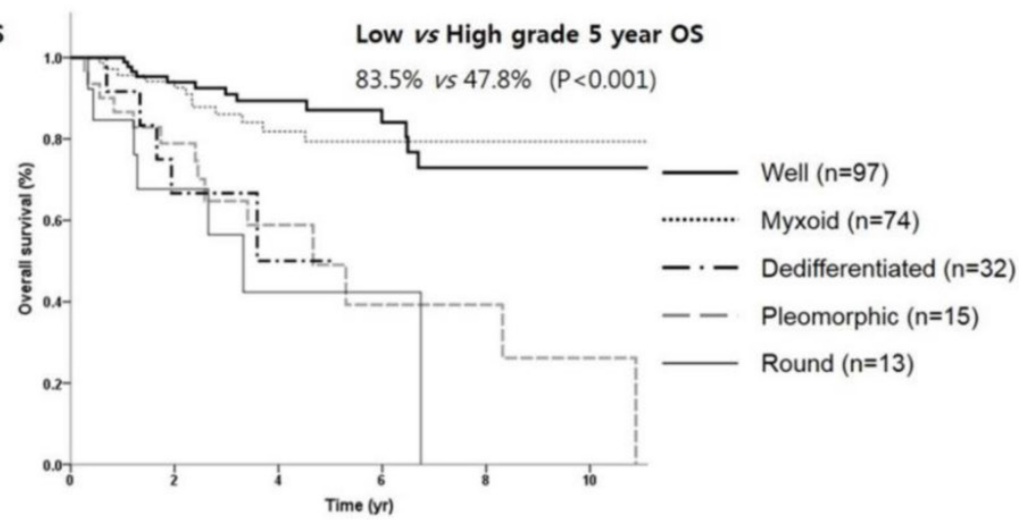

Figure 1. Survival outcome according to histologic subtypes. (A) DFS and (B) OS.

(A)

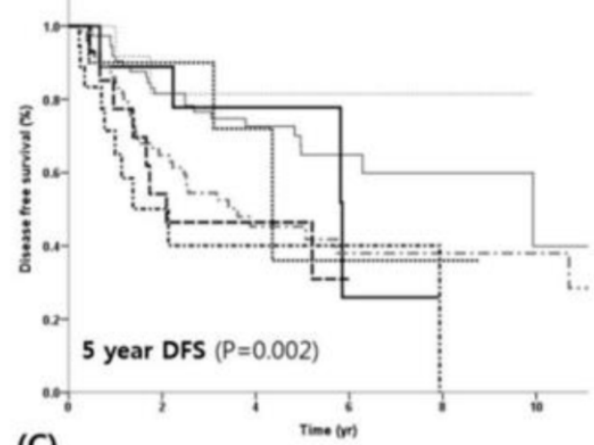

(C)

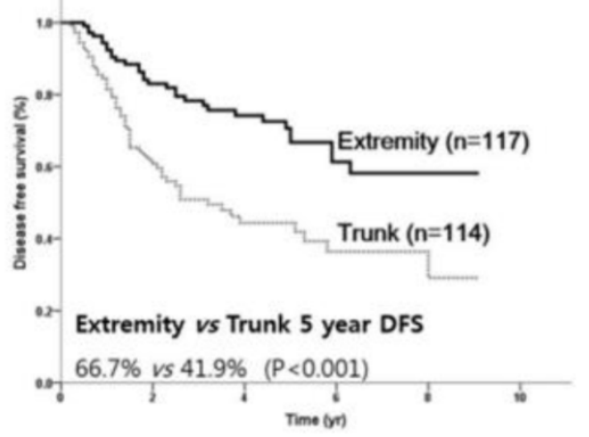

(B)

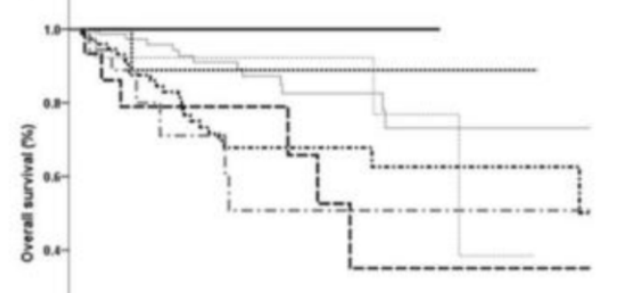

- Upper extremity $(n=11)$ Head \& Neck $(n=10)$ Lower extremity ( $n=82$ ) - - Retroperitoneum ( $n=79$ - - Thoracic cavity $(n=20)$ Inguinal \& Genital ( $n=14)$ - - Intra-abdomen ( $n=15$ )

5 year OS $(P=0.009)$

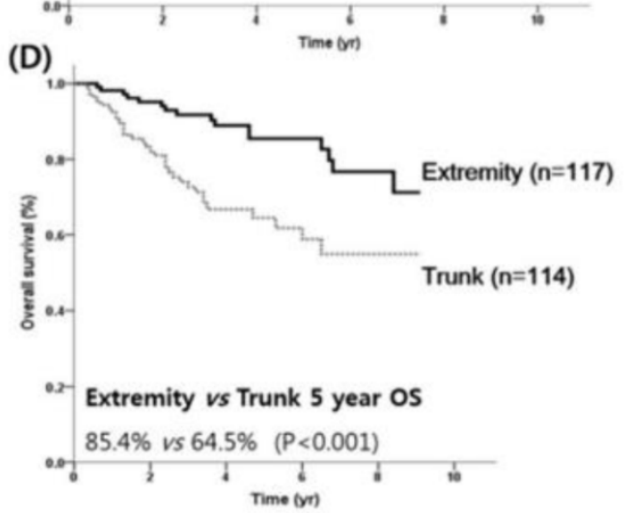

Figure 2. Survival outcome according to primary site. DFS (A) and OS (B) for each primary site. DFS (C) and OS (D) for the trunk and extremity sites. 
(A)

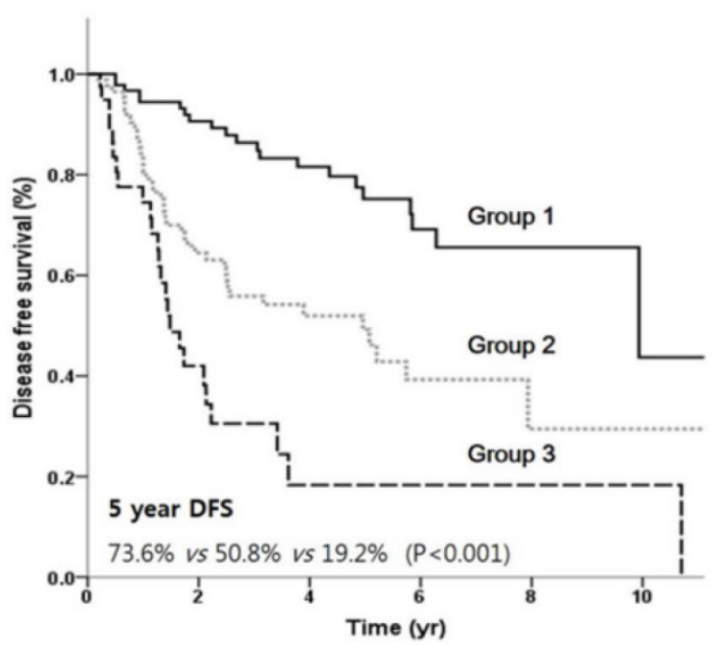

(B)

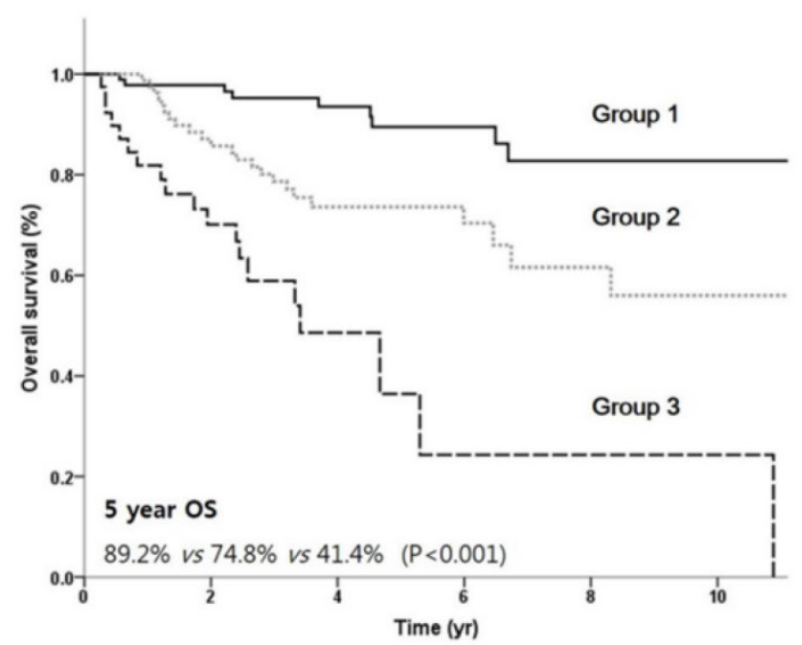

Figure 3. DFS (A) and OS (B) by prognostic model. Group 1, no risk factor; group 2, one risk factor, group 3, two risk factors.

Using the independent prognostic factors (histologic grade and primary site), prognostic grouping was performed according to the following criteria: group $1(\mathrm{n}=98)$, no risk factors; group $2(\mathrm{n}=$ 92), one risk factor; and group $3(n=41)$, two risk factors. Kaplan-Meier survival curves according to the prognostic index are shown in Figure 3. The prognostic model separated patients into three risk groups with significantly different survival outcomes. The 5-year DFS rates for groups 1, 2, and 3 were $73.6 \%, 50.8 \%$, and $19.2 \%$, respectively (Figure $3 \mathrm{~A}$ ). The 5 -year OS rates for groups 1,2 , and 3 were $89.2 \%$, $74.8 \%$, and $41.4 \%$, respectively (Figure $3 \mathrm{~B}$ ).

\section{Discussion}

In the present study, we investigated the clinicopathologic characteristics of primary liposarcoma. To our knowledge, this is the most up-to-date report providing a comprehensive epidemiological and prognostic evaluation in a large Asian liposarcoma population. In addition, we established a prognostic model to facilitate risk-adapted therapeutic strategies.

Liposarcoma accounts for $\sim 20 \%$ of STS cases in adults [12]. Accumulating evidence has suggested that major histologic subtypes of liposarcoma have differing clinical outcomes. Regarding incidence, previous studies have described few specific subtypes or organ sites and most of patients enrolled in these studies were Caucasian [13-17]. Therefore, though histologic classification is considered the most relevant prognostic factor for survival, there is limited prevalence data in Asian patients. We previously reported the clinical characteristics and outcome of curatively resected liposarcoma [18]. However, this was a single-center investigation with 94 cases, therefore, in the present study, we investigated a large homogenous Korean population and conducted subgroup analysis. A previous study indicated that the liposarcoma subtype distribution in Western patients was well-differentiated (46\%), dedifferentiated $(18 \%)$, myxoid $(18 \%)$, round-cell $(10 \%)$, and pleomorphic (8\%) [19]. The present study in Korean patients demonstrated a similar frequency of histologic subtype, in which the most common types were well-differentiated followed by myxoid and dedifferentiated types.

In the present study, the DFS and OS were well stratified according to histologic subtype. Well-differentiated and myxoid type were defined as low-grade whereas dedifferentiated, round, and pleomorphic liposarcoma were considered high-grade. The 5-year DFS and OS were significantly different between the two groups. Multivariate analyses demonstrated that histology was an independent prognostic factor for survival. These findings are congruent with those of Dalal et al. [19] who evaluated 801 nomograms for liposarcoma patients and who noted similar survival outcomes to those presented here. Confirming that histologic subtype is a strong, independent, prognostic factor for survival in patients with liposarcoma.

Regarding the primary sites, nearly two-thirds of tumors were located in the lower extremities and retroperitoneum. This finding is consistent with previous Western studies demonstrating that $25-33.4 \%$ of primary lesions were retroperitoneal [19, 20]. In addition, the present study demonstrated that extremity sites had a significantly favorable DFS compared with retroperitoneal sites. Previous studies 
have considered the lower extremities to represent the groin, thigh, or leg, whereas retroperitoneal tumors were those found in the as bowel, kidney, pancreas, bladder, and uterus. However, in the present study, we defined the inguinal/genitalia as the groin, thigh, bladder, and uterus. Contrary to patients with retroperitoneal tumors, those with tumors in the inguinal/genitalia area demonstrated a favorable survival outcome. Furthermore, we also demonstrated that those with lesions in the head and neck had better survival compared to those with lesions in the trunk. The newly classified favorable subgroups (inguinal/genitalia) might have been misclassified as the poor survival group (trunk) in previous studies. Therefore, we have elucidated the prognostic role of primary tumor sites in liposarcoma with increased clarity.

To enhance risk-based prediction after curative resection, we established a prognostic model. Primary lesion site and histologic subtype were significant independent prognostic factors, thereby, we performed prognostic grouping based on a scoring system for these factors. This yielded three distinctive groupings with differing survival outcomes according to the number of risk factors present. Given the poor prognosis of patients with $>1$ risk factors, this subgroup of patients may benefit from more aggressive pre and postoperative treatments; therefore, aggressive postoperative treatment in the context of clinical trials may be needed for group 2 .

The role of adjuvant chemotherapy or radiotherapy is poorly defined in patients with liposarcoma. In a retrospective study that included 112 patients with liposarcoma who underwent perioperative radiotherapy, Zagars et al. [[21]] showed that outcome was mainly determined by histologic subtype. Patients with well-differentiated and myxoid liposarcoma achieved a local control rate of $90 \%$, whereas those with pleomorphic liposarcoma high a high incidence of local $(37 \%)$ or distant $(41 \%)$ recurrence. In the present study, the impact of adjuvant treatment was not statistically significant. For metastatic or unresectable STSs, despite first-line standard treatment using a doxorubicin-containing regimen, the median OS was dismal (12 months) [22]. In addition, gemcitabine and docetaxel are frequently used as a second-line treatment for STSs [23], although the response rate was been reported as $16 \%$, with two patients exhibiting stable disease. A potential breakthrough in the management of STSs has been the recent introduction of pazopanib [24]. However, pazopanib has shown favorable efficacy in leiomyosarcoma and synovial sarcoma, but not in liposarcoma [25]. The underlying biological mechanisms of poor response to chemotherapy or radiotherapy have not been defined, but they might partially depend on the heterogeneity of histologic subtypes. Therefore, further histology-based strategies are strongly warranted.

This is the first study to comprehensively review and describe the clinical features and prognosis of Asian liposarcoma patients. Histologic subtype and primary tumor site were independently associated with disease-specific survival. The proposed model for primary liposarcoma demonstrated distinct groups of patients with good prognostic discrimination. We believe that the findings of the present study could facilitate further prospective studies and confirm an alternative clinical decision-making process for Asian patients with liposarcoma.

\section{Acknowledgement}

This work was supported the National Research Foundation of Korea (NRF) grant funded by the Korea government (MSIP) (2015R1C1A2A01055617, Hyo Song Kim).

\section{Competing Interests}

The authors have declared that no competing interest exists.

\section{References}

1. Dodd LG. Update on liposarcoma: a review for cytopathologists. Diagnostic cytopathology. 2012; 40: 1122-31.

2. Lemeur M, Mattei JC, Souteyrand P, Chagnaud C, Curvale G, Rochwerger A. Prognostic factors for the recurrence of myxoid liposarcoma: 20 cases with up to 8 years follow-up. Orthopaedics \& traumatology, surgery \& research : OTSR. 2015; 101: 103-7.

3. Toro JR, Travis LB, Wu HJ, Zhu K, Fletcher CD, Devesa SS. Incidence patterns of soft tissue sarcomas, regardless of primary site, in the surveillance, epidemiology and end results program, 1978-2001: An analysis of 26,758 cases. Int J Cancer. 2006; 119: 2922-30.

4. Matthyssens LE, Creytens D, Ceelen WP. Retroperitoneal liposarcoma: current insights in diagnosis and treatment. Frontiers in surgery. 2015; 2: 4.

5. Gemici K, Buldu I, Acar T, Alptekin H, Kaynar M, Tekinarslan E, et al. Management of patients with retroperitoneal tumors and a review of the literature. World journal of surgical oncology. 2015; 13: 143.

6. Evans HL. Liposarcoma: a study of 55 cases with a reassessment of its classification. Am J Surg Pathol. 1979; 3: 507-23.

7. Hoffman A, Lazar AJ, Pollock RE, Lev D. New frontiers in the treatment of liposarcoma, a therapeutically resistant malignant cohort. Drug Resist Updat. 2011; 14: 52-66.

8. Matushansky I, Hernando E, Socci ND, Matos T, Mills J, Edgar MA, et al. A developmental model of sarcomagenesis defines a differentiation-based classification for liposarcomas. The American journal of pathology. 2008; 172: 1069-80

9. Guan Z, Yu X, Wang H, Wang H, Zhang J, Li G, et al. Advances in the targeted therapy of liposarcoma. OncoTargets and therapy. 2015; 8: 125-36.

10. Karadayi K, Yildiz C, Karakus S, Kurt A, Bozkurt B, Soylu S, et al. Well-differentiated abdominal liposarcoma: experience of a tertiary care center. World journal of surgical oncology. 2015; 13: 166.

11. Zhang WD, Liu DR, Que RS, Zhou CB, Zhan CN, Zhao JG, et al. Management of retroperitoneal liposarcoma: A case report and review of the literature. Oncology letters. 2015; 10: 405-9.

12. Murphey MD. World Health Organization classification of bone and soft tissue tumors: modifications and implications for radiologists. Semin Musculoskelet Radiol. 2007; 11: 201-14.

13. Asano N, Susa M, Hosaka S, Nakayama R, Kobayashi E, Takeuchi K, et al. Metastatic patterns of myxoid/round cell liposarcoma: a review of a 25-year experience. Sarcoma. 2012; 2012: 345161

14. Fiore M, Grosso F, Lo Vullo S, Pennacchioli E, Stacchiotti S, Ferrari A, et al. Myxoid/round cell and pleomorphic liposarcomas: prognostic factors and 
survival in a series of patients treated at a single institution. Cancer. 2007; 109: 2522-31.

15. Gritli S, Khamassi K, Lachkhem A, Touati S, Chorfa A, Ben Makhlouf T, et al. Head and neck liposarcomas: a 32 years experience. Auris Nasus Larynx. 2010; 37: $347-51$.

16. Huh WW, Yuen C, Munsell M, Hayes-Jordan A, Lazar AJ, Patel S, et al. Liposarcoma in children and young adults: a multi-institutional experience. Pediatr Blood Cancer. 2011; 57: 1142-6.

17. Neuhaus SJ, Barry P, Clark MA, Hayes AJ, Fisher C, Thomas JM. Surgical management of primary and recurrent retroperitoneal liposarcoma. Br J Surg. 2005; 92: 246-52.

18. Kim HS, Lee J, Yi SY, Jun HJ, Choi YL, Ahn GH, et al. Liposarcoma: exploration of clinical prognostic factors for risk based stratification of therapy. BMC Cancer. 2009; 9: 205.

19. Dalal KM, Kattan MW, Antonescu CR, Brennan MF, Singer S. Subtype specific prognostic nomogram for patients with primary liposarcoma of the retroperitoneum, extremity, or trunk. Ann Surg. 2006; 244: 381-91.

20. Linehan DC, Lewis JJ, Leung D, Brennan MF. Influence of biologic factors and anatomic site in completely resected liposarcoma. J Clin Oncol. 2000; 18: 1637-43.

21. Zagars GK, Goswitz MS, Pollack A. Liposarcoma: outcome and prognostic factors following conservation surgery and radiation therapy. Int J Radiat Oncol Biol Phys. 1996; 36: 311-9.

22. Judson I, Verweij J, Gelderblom H, Hartmann JT, Schoffski P, Blay JY, et al. Doxorubicin alone versus intensified doxorubicin plus ifosfamide for first-line treatment of advanced or metastatic soft-tissue sarcoma: a randomised controlled phase 3 trial. Lancet Oncol. 2014; 15: 415-23.

23. Maki RG, Wathen JK, Patel SR, Priebat DA, Okuno SH, Samuels B, et al. Randomized phase II study of gemcitabine and docetaxel compared with gemcitabine alone in patients with metastatic soft tissue sarcomas: results of sarcoma alliance for research through collaboration study 002 [corrected]. J Clin Oncol. 2007; 25: 2755-63.

24. van der Graaf WT, Blay JY, Chawla SP, Kim DW, Bui-Nguyen B, Casali PG, et al. Pazopanib for metastatic soft-tissue sarcoma (PALETTE): a randomised, double-blind, placebo-controlled phase 3 trial. Lancet. 2012; 379: 1879-86.

25. Yoo KH, Kim HS, Lee SJ, Park SH, Kim SJ, Kim SH, et al. Efficacy of pazopanib monotherapy in patients who had been heavily pretreated for metastatic soft tissue sarcoma: a retrospective case series. BMC Cancer. 2015; 15: 154. 